Supporting Information

\title{
Effect of the Degree of Hydrogen Bonding on Asymmetric Lamellar Microdomains in Binary Block Copolymer Blends
}

\author{
Jongheon Kwak, Sung Hyun Han, Hong Chul Moon ${ }^{+}$, Jin Kon Kim* \\ National Creative Research Initiative Center for Smart Block Copolymers, \\ Department of Chemical Engineering, Pohang University of Science and Technology, \\ Pohang, Kyungbuk 790-784, Republic of Korea \\ Victor Pryamitsyn, Venkat Ganesan \\ Department of Chemical Engineering, University of Texas, Austin, USA \\ + Present Address: Department of Chemical Engineering, University of Seoul, \\ Seoul 130-743, Republic of Korea \\ * To whom correspondence should be addressed (jkkim@postech.ac.kr)
}

\section{Characterization, SAXS profiles and TEM images of neat block copolymers}

The weight and number average molecular weight $\left(\mathrm{M}_{\mathrm{w}}\right.$ and $\left.\mathrm{M}_{\mathrm{n}}\right)$ were measured by size exclusion chromatography (SEC) using PS standards in THF solution, and the volume fraction of PHS block ( $\left.f_{\mathrm{PHS}}\right)$ was determined by $1 \mathrm{H}$ nuclear magnetic resonance spectroscopy and known density at room temperature $\left(\mathrm{PS}=1.05 \mathrm{~g} / \mathrm{cm}^{3}\right.$; PHS $\left.=1.16 \mathrm{~g} / \mathrm{cm}^{3}\right){ }^{1}$ The hydrolysis reaction was confirmed by $1 \mathrm{H}$ NMR spectra. 
(a)

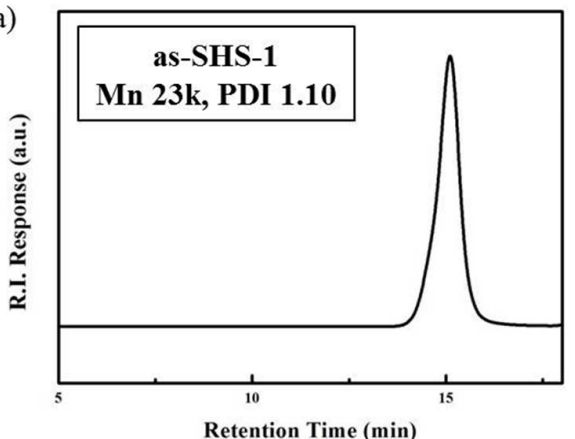

(c)

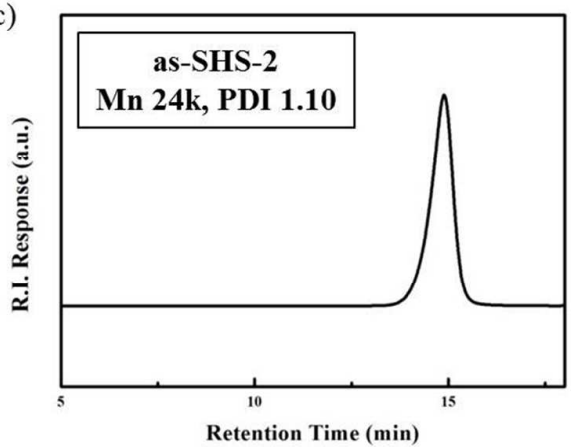

(e)

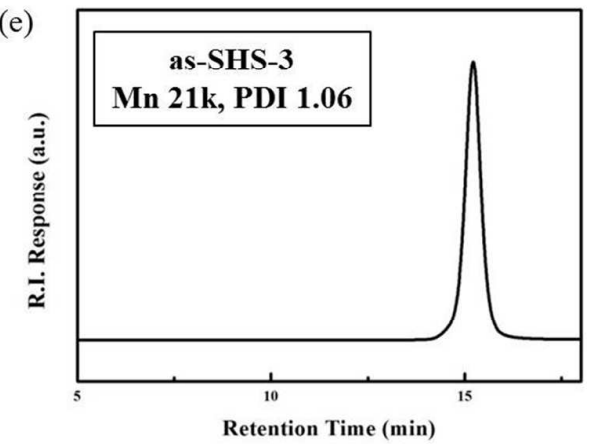

(b)

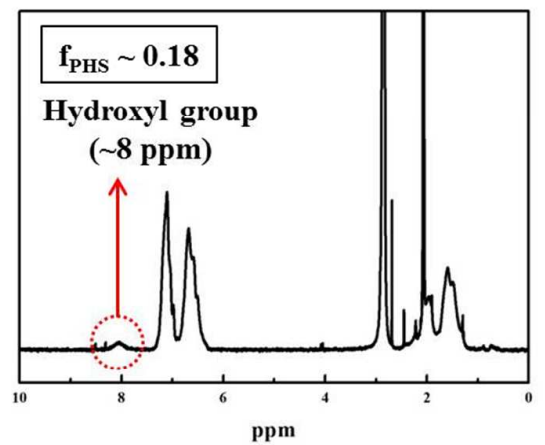

(d)

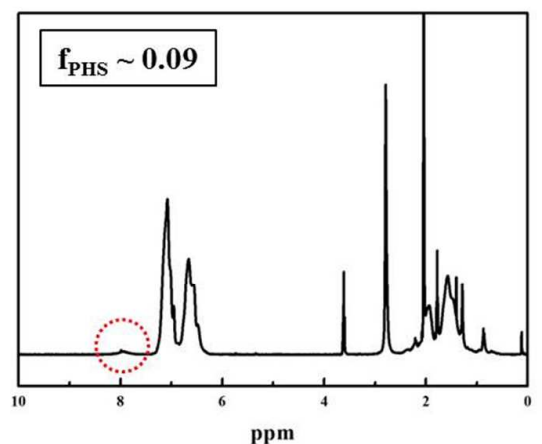

(f)

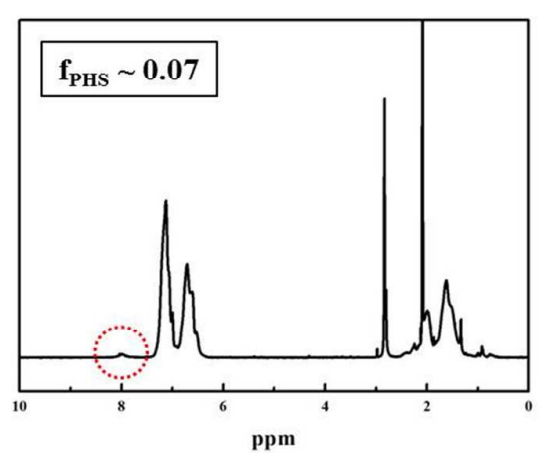

Figure S1. SEC traces (left panels) and ${ }^{1} \mathrm{H}-\mathrm{NMR}$ spectra (right panels) for as-SHS-1 (a and b), as-SHS-2 (c and d), and as-SHS-3 (e and f). 

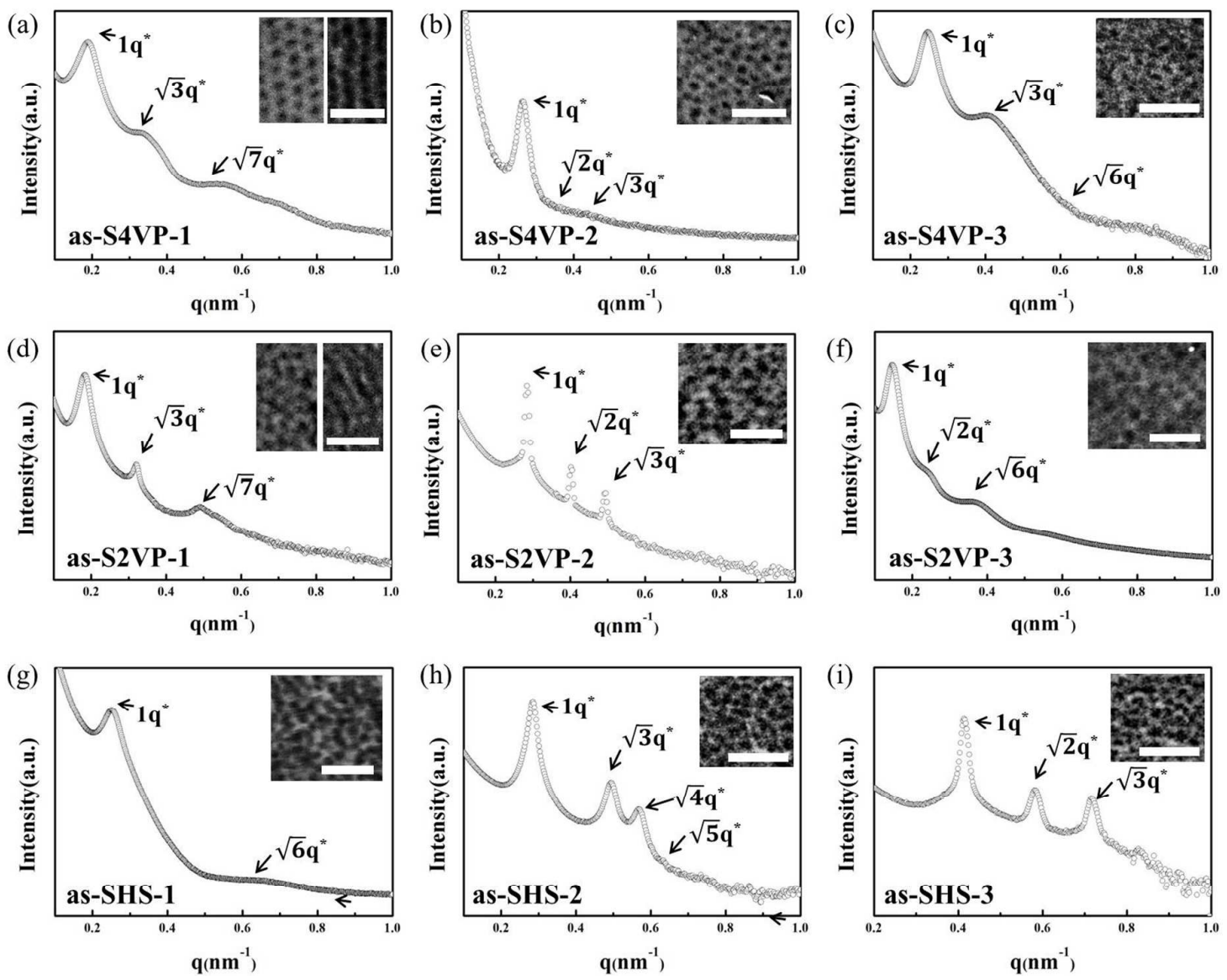

Figure S2. SAXS profiles and TEM images (inset) for neat block copolymers: (a) as-S4VP-1,

(b) as-S4VP-2, (c) as-S4VP-3, (d) as-S2VP-1, (e) as-S2VP-2 (f) as-S2VP-3, (g) as-SHS-1, (h) as-SHS-2 and (i) as-SHS-3. Scale bar in TEM image is $100 \mathrm{~nm}$.

Figure S2 gives SAXS profiles that show each characteristic scattering peaks, and TEM images for each neat block copolymer. SAXS profiles representing cylindrical microdomains are observed for both as-S4VP-1 (a) and as-S2VP-1 (d). From TEM images of crosssectional cylinders (left images) and longitudinal cylinders (right images), both samples exhibit cylindrical microdomains, consistent with the SAXS profiles. On the other hand, all other neat block copolymers (as-S4VP-2 (b), as-S4VP-3 (c), as-S2VP-2 (e), as-S2VP-3 (f), as-SHS-1 (g), as-SHS-2 (h), and as-SHS-3 (i)) show spherical microdomains from SAXS profiles and TEM images. 


\section{SAXS profiles of binary blends}

Figure S3 shows SAXS profiles for 50/50 (wt/wt) as-S4VP-1/as-SHS-1 blend (Figure S3a), 50/50 (wt/wt) as-S2VP-1/as-SHS-1 blend (Figure S3b) and 50/50 (wt/wt) as-S2VP-3/asSHS-1 blend (Figure S3c). Three SAXS profiles show the scattering peaks at the position of $1: 2: 3$ relative to $\mathrm{q}^{*}$, indicating that all blends have lamellar microdomains.
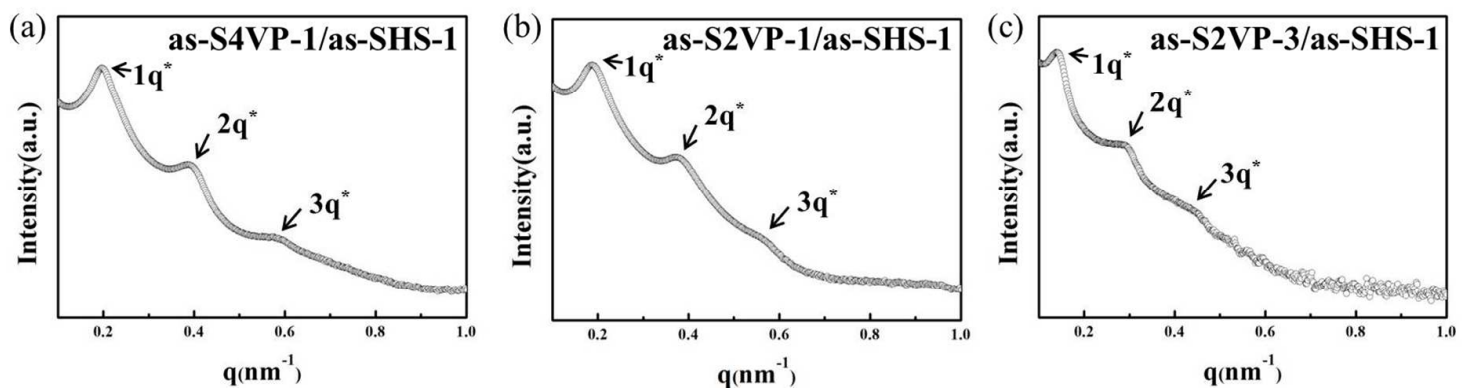

Figure S3. SAXS profiles for (a) 50/50 (wt/wt) as-S4VP-1/as-SHS-1 blend, (b) 50/50 (wt/wt) as-S2VP-1/as-SHS-1 blend and (c) 50/50 (wt/wt) as-S2VP-3/as-SHS-1 blend. 


\section{Fitting SAXS profile with a variable lamellar thickness structure model. ${ }^{2,3}$}

Sharp peaks in SAXS profiles expected from the ideal two-phase lamellar system are broadened and reduced in height due to some imperfections. Thus, to obtain more accurate results, SAXS profiles should be analyzed based on variable lamellar thickness model (Figure S4a), where the thickness of the lamellae changes more or less randomly from one to the next. This model assumes the thickness of phase $A$ (or $B$ ) is distributed as per the probability $\mathrm{P}_{\mathrm{a}}(\mathrm{a}) d a$ (or $\mathrm{P}_{\mathrm{b}}(\mathrm{b}) d b$ ) of finding it between $a$ and $a+d a$ (or $\left.b+d b\right)$. Furthermore, it assumes that there is no correlation between the thicknesses of neighboring lamellae. The lamellar thickness distributions $\left(\mathrm{P}_{\mathrm{a}}(\mathrm{a})\right.$ and $\left.\mathrm{P}_{\mathrm{b}}(\mathrm{b})\right)$ are assumed to become the Gaussian distribution:

$$
P_{a}(a)=\frac{1}{\sqrt{2 \pi} \sigma_{a}} \exp \left[-\frac{\left(a-d_{a}\right)^{2}}{2 \sigma_{a}^{2}}\right]
$$

where $d_{a}$ and $\sigma_{\mathrm{a}}$ are the average and the standard deviation of $a$. A similar expression is assumed for $P_{b}(b)$. After a long calculation, finally for small values of $\sigma_{\mathrm{a}}$ and $\sigma_{\mathrm{b}}$ the relative height of the peak of the successive order $n$ can be estimated as follows:

$$
I_{1}(q)=16 N \frac{(\Delta \rho)^{2}}{q^{4}} \frac{\sigma_{b}^{2} \sin ^{2}\left(\frac{q d_{a}}{2}\right)+\sigma_{a}^{2} \sin ^{2}\left(\frac{q d_{b}}{2}\right)}{\left(\sigma_{a}^{2}+\sigma_{b}^{2}\right)^{2}+\left(\frac{16}{q^{4}}\right) \sin ^{2}\left(\frac{q d}{2}\right)}
$$

The denominator of Eqn. (2) means that peaks are located near the q values, which makes $\sin ^{2}(q d / 2)$ equal to zero: $q=2 \pi n / d$. The exact locations of the maximum are slightly shifted from these $q$ values because of the distortion to the peak shape imparted by the numerator of Eqn. (2).

We predicted the volume fraction of PS microdomain in binary blend of 50/50 (wt/wt) asS4VP-2/as-SHS-1 via the intensity function $I_{l}(q)$, which was curve-fitted from Eqn. (2) (Figure S4b). The predicted $f_{P S}$ is 0.86 . 


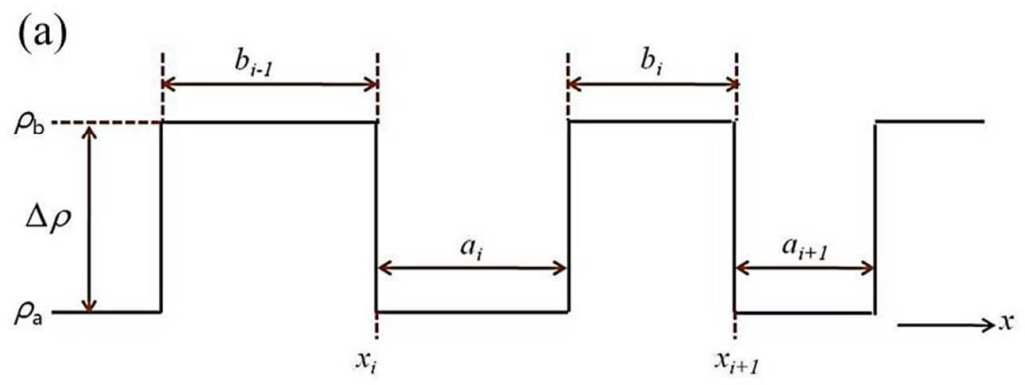

(b)

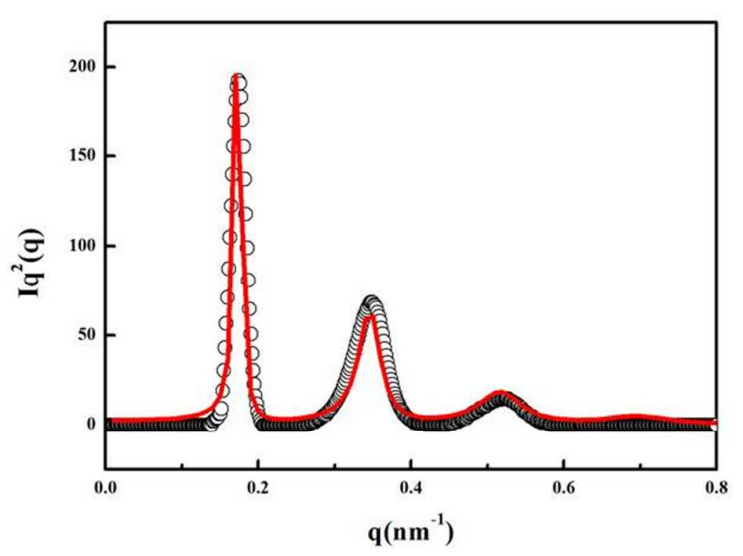

Volume fraction of $\operatorname{PS}\left(\phi_{\mathrm{PS}}\right)=\mathbf{0 . 8 6}$

$\mathrm{L}_{0}=36 \mathrm{~nm}\left(\mathrm{~d}_{\mathrm{a}}=31 \mathrm{~nm}, \mathrm{~d}_{\mathrm{b}}=5.0 \mathrm{~nm}\right)$

$\left(\sigma_{\mathrm{a}}=2.6 \mathrm{~nm}, \sigma_{\mathrm{b}}=2.1 \mathrm{~nm}\right)$

Figure S4. (a) Scattering length density profile in the direction perpendicular to the lamellar plane where each lamellar microdomain has different thicknesses. (b) Curve-fitted SAXS profiles of 50/50 (wt/wt) as-S4VP-2/as-SHS-1 blend by using the variable lamellar thickness model.

\section{References}

(1) Han, S. H.; Kim, J. K.; Pryamitsyn, V.; Ganesan, V. Macromolecules 2011, 44, 49704976.

(2) Roe, R-. J-. Methods of X-ray and Neutron Scattering in Polymer Science, Oxford University Press 2000. 
(3) Kwak, J.; Han, S. H.; Moon, H. C.; Kim, J. K.; Koo, J.; Lee, J-.; Pryamitsyn, V.; Ganesan, V. Macromolecules 2015, 48, 1262-1266. 